\title{
The polymorphisms of extracellular matrix-remodeling genes are associated with pelvic organ prolapse
}

\author{
Lei $\mathrm{Li}^{1} \cdot$ Yidi Ma ${ }^{1} \cdot$ Hua Yang ${ }^{1} \cdot$ Zhijing Sun $^{1} \cdot$ Juan Chen ${ }^{1} \cdot$ Lan Zhu ${ }^{1}$ (D)
}

Received: 7 April 2021 / Accepted: 10 June 2021 / Published online: 1 January 2022

(C) The Author(s) 2021

\begin{abstract}
Introduction and hypothesis Extracellular matrix (ECM) synthesis and metabolism abnormalities may influence the pelvic supporting system and lead to the occurrence and development of pelvic organ prolapse (POP). Genetic polymorphisms of such related genes have been increasingly studied. This study aims to explore the association between the single-nucleotide polymorphisms (SNPs) of genes encoding ECM processing enzymes (a disintegrin and metalloproteinase with thrombospondin motifs [ADAMTSs]), ECM degrading enzymes (matrix metalloproteinases [MMPs]) and their tissue inhibitors of metalloproteinase (TIMPs), and POP.

Methods We conducted an association study including 48 women with POP at stages III and IV and 48 women without prolapse in Chinese groups. SNPs were identified using the target region sequencing technique. We performed Fisher's exact tests to assess the association between SNPs and POP in the unadjusted model and logistic regression analysis in the adjusted model, adjusting for delivery and pregnancy.

Results There was a significant association between TIMP2 SNP rs2277698 (odds ratio [OR], 0.37; 95\% confidence interval [CI], 0.16-0.82; $P=0.015$ ), ADAMTS13 SNP rs149586801 (OR, 0.18; 95\% CI, 0.05-0.69; $P=0.012$ ), and ADAMTS1 SNPs rs370850 and rs422803 (OR, 3.71; 95\% CI, 1.35-10.15; $P=0.011$ for both), rs402007, rs428785, rs434857, and rs445784 (OR, 2.18; $95 \% \mathrm{CI}, 1.05-4.56 ; P=0.038$ for the four), and POP in the adjusted model.

Conclusion TIMP2, ADAMTS13, and ADAMTS1 might be candidate genes for POP. Our results provide preliminarily new evidence for future investigation of these genes in the pathophysiology of POP.
\end{abstract}

Keywords Pelvic organ prolapse $\cdot$ Single-nucleotide polymorphism - Extracellular matrix synthesis and metabolism . ADAMTSs $\cdot$ MMPs $\cdot$ TIMPs

\section{Introduction}

Pelvic organ prolapse (POP) is a common pelvic disorder among older women. It is characterized as the downward bulging of uterus, bladder, rectum, etc., urinary and fecal inconvenience, and sexual dysfunction, which negatively affect women's quality of life and social activities [1]. The prevalence of symptomatic POP in China for postmenopausal

Lan Zhu

zhu_julie@vip.sina.com

$1 \quad$ National Clinical Research Center for Obstetric \& Gynecologic Diseases, Department of Obstetrics and Gynecology, Peking Union Medical College Hospital, Chinese Academy of Medical Sciences \& Peking Union Medical College, No. 1 Shuai Fu Yuan, Eastern District, Beijing 100730, China women is $15 \%$ [2]. The lifetime risk of women undergoing POP surgery is $11-19 \%$, and $30 \%$ patients still need a repeat operation [3]. Therefore, POP is becoming a social health and family burden. Understanding the pathogenesis of POP is necessary for developing novel prevention and intervention strategies for the clinic in the future.

The etiology of POP is complicated. Race/ethnicity, advancing age, obesity, higher parity, and menopause are known risk factors for POP. In addition, it is well demonstrated that $\mathrm{POP}$ has the characteristics of familial aggregation, as the risk of POP in women increases if their mothers or sisters have suffered from the disease, indicating a genetic contribution to POP [3]. Previous genetic analyses have revealed candidate genes associated with POP. These genes included collagen type I alpha (COL1A1) $[4,5]$, collagen type III alpha 1 (COL3A1) [6], laminin gamma-1 (LAMC1) [7], and matrix metalloproteinases $(M M P s)$ [8], which are all enrolled in 
extracellular matrix (ECM) pathways. The latest review comprehensively summarized and identified genetic polymorphisms associated with POP, including steroid hormone receptor genes and collagen/elastic fiber synthesis genes [5]. Thus, ECM synthesis and metabolism abnormalities may affect the pelvic supporting system and have been considered the potential causes for POP.

MMPs are a family of multiple catabolic proteases involved in the degradation of collagen fibers and other components of ECM. Several previous studies have identified the existence of polymorphisms in the promoter regions of $M M P 1,3$, and 9 genes, which could alter the expression of these genes and increase the risk of POP [8-11]. Chen et al. [12] detected three MMP9 SNPs (rs3918242, rs17576, and rs2250889) and found rs17576 to be associated with POP. Wu et al. [13] further assessed eight MMP9 SNPs in an association study and found two SNPs, rs3918253 and rs3918256, associated with prolapse. Wang et al. [14] demonstrated that MMP10 SNP rs17435959 was associated with POP in a Chinese group of 91 cases and 172 controls. Besides these limited studies, other MMP members have not been studied in POP.

The bioactivity of MMPs is regulated by tissue inhibitors of metalloproteinase (TIMPs), which are also crucial factors of ECM remodeling. Allen-Brady et al. [15] carried out a genome-wide linkage study from 225 familial POP cases and found significant linkage on chromosome 17q25. In this region, the most highlighted gene for POP is TIMP2, which has been considered to be involved in connective tissue disorders and vascular diseases.

ADAMTSs, known as a disintegrin and metalloproteinase with thrombospondin motif, are a family of proteinases involved in procollagen processing. They can cleave the Nterminal of the peptide chains of procollagen molecules, which are the precursors of mature collagen [16]. Thus, ADAMTSs participate in the synthesis of ECM. Alarab et al. [17] found that ADAMTS2 was increased in the vaginal tissues of POP patients. However, there has been no research focusing on the relationship between the polymorphisms of TIMPs/ADAMTSs and POP.

As the fine balance between the synthesis and degradation of ECM components is essential to the integrity of the pelvic floor supportive structures, and given the previous limited findings on some of the MMP studies, and also given the fact that there has been no research on the association between the single-nucleotide polymorphisms (SNPs) of TIMPs/ ADAMTSs and risks of POP, we have carried out a casecontrol association study in a group of Chinese women. We supposed that besides $M M P 1,3,9$, and 10, SNPs from other MMP members, TIMPs, and ADAMTSs would be associated with POP. Using a target region sequencing technique, we (1) confirmed the previous findings on MMPs and (2) investigated the susceptible loci of some of the other MMP, ADAMTS, and TIMP family genes for POP. We believe that these results might offer novel insights into the molecular mechanisms of POP development in Chinese women.

\section{Materials and methods}

\section{Study samples}

This was part of a case-control association study in which we recruited women from Peking Union Medical College Hospital (PUMCH) in Beijing from October 2016 to May 2017. Cases were POP patients diagnosed at Pelvic Organ Prolapse Quantification (POP-Q) stages III and IV from the Department of Gynecology and Obstetrics, and control women were diagnosed with no prolapse and have not suffered from prolapse surgery from the Physical Examination Center. All the participants signed the informed consents, and the study was approved by the Ethics Committee of PUMCH.

We excluded women with the well-known connective tissue diseases, including Marfan syndromes, Ehlers-Danlos syndromes, rheumatoid arthritis or scleroderma, and women with neurological diseases including sclerosis or stroke. As the genetic variants vary between different race/ethnic groups, all the samples were limited to Chinese ancestry. Finally, we recruited 48 cases and 48 controls subjected to the next genotyping. Sociodemographic data and physical information were collected as summarized in our previous study [7].

\section{Genotyping and SNP selection}

Genome DNA was extracted from the peripheral venous whole-blood samples using a Puregene Blood Kit (QIAGEN, Hilden, Germany). The target region sequencing approach was used to achieve a comprehensive assessment of the specific genes; $1-2 \mu \mathrm{g}$ DNA of each blood sample was sequenced by Agilent Liquid Capture System (Agilent SureSelect Custom Kit; Agilent Technologies, Palo Alto, CA, USA) at Novogene (Novogene Co., Ltd., Beijing, China) based on the Illumina HiSeq 4000 platform to provide a more than $200 \times$ deep of sequencing.

The valid sequencing data landed in a BAM file by Burrows-Wheeler Aligner (BWA) software based on the reference genome (UCSC hg19). Samtools and Picard (http:// broadinstitute.github.io/picard) were used to sort the files and mark the duplicated reads. Samtools mpileup and bcftools were used to call variants, insertions, and deletions. The SNP quality control included SNPs with read depth $>4$, mapping quality $>30$, and the variant quality $>20$. Variants with a minor allele frequency (MAF) $>5 \%$ remained. SNPs were assessed by PolyPhen-2, SIFT, MutationTaster, and CADD software, respectively, for the prediction of their 
functional effects. ANNOVAR was applied to provide the annotations of the position, type, and conservative prediction of the altered alleles and other information.

\section{Statistical analysis}

We respectively conducted an unadjusted model and adjusted model to investigate the associations between SNPs and POP. Fisher's exact tests were used for the unadjusted model, and logistic regression models were used for the adjusted model via PLINK. The multivariable models were adjusted for pregnancy and delivery as we did not match the two variables between case and control groups.

Odds ratio (OR) and $95 \%$ confidence interval (CI) were shown for each of the variants with $\mathrm{OR}<1$ indicating a protective effect and OR $>1$ indicating a risk effect [18]. $P<0.05$ was considered significant and $<0.1$ to be suggestively significant. Hardy-Weinberg equilibrium was assessed using the $x^{2}$ test. The schematic diagrams of the gene structure that showed exons and introns as well as the type of the SNPs were graphed by the Exon-Intron Graphic Maker (http://wormweb. org/exonintron). The linkage disequilibrium (LD) graphs indicating $r^{2}$ between SNPs were drawn by Haploview (Harvard Broad Institute, Boston, MA, USA).

\section{Results}

We have described sample characteristics in our previous study. Briefly, we matched age $(61.85 \pm 10.66$ vs. $62.73 \pm$ 8.88 years, $P=0.663)$ and body mass index (BMI) $(25.11 \pm$ 3.83 vs. $24.52 \pm 3.48 \mathrm{~kg} / \mathrm{m}^{2}, P=0.434$ ) between case and control groups. However, the case group had approximately $\geq 1$ delivery $(2.35 \pm 1.34$ vs. $1.58 \pm 1.06, P=0.005)$ and pregnancy ( $3.60 \pm 1.89$ vs. $2.50 \pm 1.50, P=0.023)$ in contrast to the control group. In the case group, 41 women $(85.42 \%)$ were diagnosed at POP-Q stage III and 7 women (14.58\%) were at stage IV as previously described [7].

Since the synthesis and degradation of the ECM is a dynamic process for the pelvic tissues, in this study, we considered several of the enzymes related to ECM processing (ADAMTS1, 2, 3, 8, 13, 14) and degradation (MMP1, 2, 3, 8, 9, 10, 13, and TIMP1, 2, 3, 4) in Chinese women. These genes are listed in Table 1. We have analyzed the association between SNPs and POP in both the unadjusted and adjusted model with adjustment for delivery and pregnancy. Among all these genes, we have genotyped five SNPs with $P$ values $<0.1$ in the unadjusted model and 16 SNPs with $P$ values $<0.1$ in the adjusted model that were considered to be significant or suggestively significant (Table S1). These SNPs were additionally described in detail in Table 2 and were all in HardyWeinberg equilibrium as they reached $10^{-6}[19]$.
In the analysis of the MMP family genes, we have identified a trend toward significance for MMP9 SNP rs3918254 and POP (OR, 2.21; 95\% CI, 0.91-5.36; $P=0.079)$ after the adjustment, which was an intronic SNP. For other MMP members, we have identified two new MMP13 SNPs, rs3758853 and rs78356340, which were intronic SNPs, which showed a suggestively significant association with POP in the adjusted model (for both, OR, 3.02; 95\% CI, 0.91-9.99; $P=$ 0.070) (Table 2).

In the analysis of TIMP family genes, TIMP2 SNP rs2277698 showed a significant association with POP in the adjusted model (OR, 0.37 ; 95\% CI, 0.16-0.82; $P=0.015)$ and a suggestively significant association with $\mathrm{POP}$ in the unadjusted model (OR, 0.50; 95\% CI, 0.25-0.98; $P=0.062$ ). TIMP3 SNP rs9862 showed a suggestively significant association with POP in the adjusted model (OR, 1.69; 95\% CI, 0.92-3.13; $P=0.093$ ). rs 2277698 and rs 9862 were synonymous SNPs that did not result in amino acid changes. TIMP4 SNP rs10433537 showed a suggestively significant association with POP in both the unadjusted and adjusted model (OR, 4.83; 95\% CI, 1.02-23.13; $P=0.058$ and OR, 4.16 ; 95\% CI, $0.85-20.31 ; P=0.078$, respectively), which was an intronic SNP (Table 2).

For the analysis of some of the ADAMTS family genes, there was a significant association between ADAMTS13 SNP rs149586801, which was an intronic SNP, and POP in both the unadjusted and adjusted models (OR, 0.26 ; $95 \%$ CI, $0.07-$ $0.78 ; P=0.011$ and $\mathrm{OR}, 0.18 ; 95 \% \mathrm{CI}, 0.05-0.69 ; P=0.012$, respectively). There was also a suggestively significant association between rs 1055432 and POP in the adjusted model (OR, 0.48; 95\% CI, 0.22-1.09; $P=0.080$ ). rs 1055432 was a synonymous SNP that did not lead to the amino acid alteration (Table 2). We have also identified a trend toward significance for the ADAMTS14 SNP rs4747097 and POP (OR, 1.83; 95\% CI, $0.93-3.56 ; P=0.078)$ in the adjusted model, which was an intronic SNP (Table 2).

In the analysis of ADAMTS1, rs370850 and rs422803, which are intronic SNPs, were respectively significantly associated with POP in the adjusted model (for both, OR, 3.71; 95\% CI, 1.35-10.15; $P=0.011$ ). They also showed a trend toward significance with POP in the unadjusted model (for both, OR, 1.87; 95\% CI, 0.94-3.77; $P=0.076$ ). rs402007, rs428785, rs434857, and rs445784 were respectively significantly associated with POP in the adjusted model (for all, OR, $2.18 ; 95 \%$ CI, $1.05-4.56 ; P=0.038)$. rs 402007 was in the 5'UTR region of the ADAMTS1 gene. rs428785 was a missense SNP. rs434857 and rs445784 were synonymous SNPs that did not lead to amino acid changes. There was also a trend toward significance for the SNP rs436525 and POP in the adjusted model (OR, 1.86; 95\% CI, 0.91-3.82; $P=0.090$ ). rs436525 was also a synonymous SNPs without amino acid changes (Table 2). These seven SNPs are indicated in the schematic of Fig. 1A where the exons and introns of the 
Table 1 Genes genotyped in this study

\begin{tabular}{|c|c|c|c|}
\hline Gene & Full name & cytoBand & Description \\
\hline$M M P 1$ & Matrix metallopeptidase 1 & $11 \mathrm{q} 22.2$ & Interstitial collagenase, and cleaves collagen I, II, and III \\
\hline$M M P 2$ & Matrix metallopeptidase 2 & $16 \mathrm{q} 12.2$ & Gelatinase A, and cleaves collagen I, II, III, IV and V, and elastin \\
\hline$M M P 3$ & Matrix metallopeptidase 3 & $11 \mathrm{q} 22.2$ & $\begin{array}{l}\text { Stromelysin-1, and cleaves collagen III, IV, IX and X, laminin, and } \\
\text { cartilage proteoglycans }\end{array}$ \\
\hline$M M P 8$ & Matrix metallopeptidase 8 & $11 \mathrm{q} 22.2$ & Interstitial collagenase, and cleaves collagen I, II, and III \\
\hline$M M P 9$ & Matrix metallopeptidase 9 & $20 \mathrm{q} 13.12$ & Gelatinases B, and cleaves collagen IV and V \\
\hline MMP10 & Matrix metallopeptidase 10 & $11 \mathrm{q} 22.2$ & $\begin{array}{l}\text { Stromelysins-2, and cleaves laminin, elastin and fibronectin, however, } \\
\text { weakly collagens }\end{array}$ \\
\hline$M M P 13$ & Matrix metallopeptidase 13 & $11 \mathrm{q} 22.2$ & Interstitial collagenase, and cleaves collagen I, II and III, especially type II \\
\hline $\begin{array}{l}\text { TIMP1 } \\
\text { TIMP2 }\end{array}$ & $\begin{array}{l}\text { Tissue inhibitor of metalloprotease } 1 \\
\text { Tissue inhibitor of metalloprotease } 2\end{array}$ & $\begin{array}{l}\mathrm{Xp} 11.3 \\
17 \mathrm{q} 25.3\end{array}$ & Specific inhibitors of MMPs, involved in the degradation of ECM \\
\hline TIMP3 & Tissue inhibitor of metalloprotease 3 & $22 q 12.3$ & \\
\hline TIMP4 & Tissue inhibitor of metalloprotease 4 & $3 \mathrm{p} 25.2$ & \\
\hline ADAMTS1 & $\begin{array}{l}\text { ADAM metallopeptidase with thrombospondin } \\
\text { type } 1 \text { motif } 1\end{array}$ & $21 \mathrm{q} 21.3$ & $\begin{array}{l}\text { Aggrecanase, can degrade aggrecan, a cartilage proteoglycan, and can } \\
\text { activate metalloproteases }\end{array}$ \\
\hline ADAMTS2 & $\begin{array}{l}\text { ADAM metallopeptidase with thrombospondin } \\
\text { type } 1 \text { motif } 2\end{array}$ & $5 \mathrm{q} 35.3$ & $\begin{array}{l}\text { Cleaves the } \mathrm{N} \text {-terminal propeptides from the fibrillar procollagens I and II } \\
\text { as well as excises lysyl oxidase }\end{array}$ \\
\hline ADAMTS3 & $\begin{array}{l}\text { ADAM metallopeptidase with thrombospondin } \\
\text { Type } 1 \text { motif } 3\end{array}$ & $4 \mathrm{q} 13.3$ & Cleaves the N-terminal propeptides from the fibrillar procollagen II \\
\hline ADAMTS8 & $\begin{array}{l}\text { ADAM metallopeptidase with thrombospondin } \\
\text { Type } 1 \text { motif } 8\end{array}$ & $11 \mathrm{q} 24.3$ & Inhibits angiogenesis \\
\hline ADAMTS13 & $\begin{array}{l}\text { ADAM metalloproteinase with thrombospondin } \\
\text { Type } 1 \text { motif } 13\end{array}$ & $9 \mathrm{q} 34.2$ & Cleaves von Willebrand Factor \\
\hline ADAMTS14 & $\begin{array}{l}\text { ADAM metallopeptidase with thrombospondin } \\
\text { Type } 1 \text { motif } 14\end{array}$ & $10 \mathrm{q} 22.1$ & Cleaves the N-terminal propeptides from the procollagen I \\
\hline
\end{tabular}

$M M P$ matrix metalloproteinase, TIMP tissue inhibitors of metalloproteinase, ADAMTS a disintegrin and metalloproteinase with thrombospondin motif, $E C M$ extracellular matrix

ADAMTS1 gene were described. rs436525 was in good LD with six other SNPs $\left(r^{2}>0.8\right)$, and rs370850, rs422803, rs402007, rs428785, rs434857, and rs445784 were in perfect LD between each other $\left(\mathrm{r}^{2}=1\right)$ (Fig. 1B). The missense SNP rs428785 resulted in a substitution of alanine to proline, which might cause the change of the protein secondary structure.

\section{Discussion}

POP is a common female pelvic disorder due to the decline of the pelvic floor supportive tissues. The imbalance of synthesis and degradation of collagen and other ECM components plays a role in the pathogenesis of POP. Such regulation of ECM stability mainly relied on MMPs, which can cleave collagen, elastin, and proteoglycans, etc., and their inhibitors TIMPs [20]. ADAMTSs are enzymes involved in the process of procollagen [16]. Thus, many studies have focused on these ECM remolding genes. In this case-control association study in Chinese women, we sequenced some of the MMP, TIMP, and ADAMTS family genes. To test the association of SNPs and POP, we performed Fisher's exact tests in the unadjusted model. As we matched case and control groups by age, BMI, and the number of postmenopausal women, and did not match for delivery and pregnancy, which were also crucial risk factors for POP and might be the potential to introduce bias, we additionally conducted logistic regression analysis in the model adjusted for delivery and pregnancy. We tested the results of previous studies on $M M P 9$ and 10 polymorphisms and further analyzed the association of some other MMP genes, TIMPs, and some ADAMTS genes with risks of POP for the first time. Besides $M M P 9$ and 10, our study provided novel evidence that MMP13, TIMP2, 3, 4, and ADAMTS1, 13, 14 might be possible candidate genes for POP.

MMPs act on ECM breakdown in multiple physiological and pathological processes, including embryonic development, tissue remodeling, wound repair, angiogenesis, inflammatory processes, and tumor progression [21]. According to the structure of the domain and the type of their substrates, MMPs, with 24 family members in mammals, are classified into several subtypes [22]. Among those, MMP1, MMP8, and MMP13 are interstitial collagenases, which mainly degrade collagens I, II, and III. MMP2 and MMP9 are gelatinases, which can cleave collagens and gelatins [23]. MMP2 can also degrade collagens I, II, and III [24]. MMP3 and MMP10 are stromelysins. In addition to degrading ECM components, 
Table 2 Analysis for both unadjusted and adjusted models for some of the MMP, TIMP, and ADAMTS family genes

\begin{tabular}{|c|c|c|c|c|c|c|c|c|c|}
\hline \multirow[t]{2}{*}{ SNP } & \multirow[t]{2}{*}{ Allele frequency } & \multicolumn{2}{|c|}{ Unadjusted } & \multicolumn{2}{|c|}{ Adjusted $^{\mathrm{a}}$} & \multirow[t]{2}{*}{ HWE } & \multirow[t]{2}{*}{ Function } & \multirow[t]{2}{*}{ Exonic function } & \multirow[t]{2}{*}{ AA change } \\
\hline & & $P$ value & OR $(95 \% \mathrm{CI})$ & $P$ value & OR $(95 \% \mathrm{CI})$ & & & & \\
\hline \multicolumn{10}{|l|}{$M M P 9$} \\
\hline rs3918254 & $\mathrm{T}(0.20)$ & 0.163 & $1.90(0.80-4.72)$ & 0.079 & $2.21(0.91-5.36)$ & 0.454 & Intronic & - & - \\
\hline \multicolumn{10}{|l|}{$M M P 13$} \\
\hline rs3758853 & $\mathrm{G}(0.11)$ & 0.104 & $2.96(0.84-13.24)$ & 0.070 & $3.02(0.91-9.99)$ & 0.447 & Intronic & - & - \\
\hline rs 78356340 & $\mathrm{~A}(0.11)$ & 0.104 & $2.96(0.84-13.24)$ & 0.070 & $3.02(0.91-9.99)$ & 0.447 & Intronic & - & - \\
\hline \multicolumn{10}{|l|}{ TIMP2 } \\
\hline rs 2277698 & $\mathrm{~T}(0.18)$ & 0.062 & $0.50(0.25-0.98)$ & 0.015 & $0.37(0.16-0.82)$ & 0.781 & Exonic & Synonymous & S101S \\
\hline \multicolumn{10}{|l|}{ TIMP3 } \\
\hline rs9862 & $\mathrm{C}(0.71)$ & 0.130 & $1.66(0.91-3.03)$ & 0.093 & $1.69(0.92-3.13)$ & 0.367 & Exonic & Synonymous & $\mathrm{H} 83 \mathrm{H}$ \\
\hline \multicolumn{10}{|l|}{ TIMP4 } \\
\hline rs 10433537 & $\mathrm{~T}(0.09)$ & 0.058 & $4.83(1.02-23.13)$ & 0.078 & $4.16(0.85-20.31)$ & 0.262 & Intronic & - & - \\
\hline \multicolumn{10}{|l|}{ ADAMTS1 } \\
\hline rs 370850 & $\mathrm{~T}(0.41)$ & 0.076 & $1.87(0.94-3.77)$ & 0.011 & $3.71(1.35-10.15)$ & 0.00019 & Intronic & - & - \\
\hline rs422803 & A $(0.41)$ & 0.076 & $1.87(0.94-3.77)$ & 0.011 & $3.71(1.35-10.15)$ & 0.00019 & Intronic & - & - \\
\hline rs402007 & $\mathrm{G}(0.47)$ & 0.106 & $1.68(0.90-3.14)$ & 0.038 & $2.18(1.05-4.56)$ & 0.140 & UTR5 & - & - \\
\hline rs 428785 & $\mathrm{G}(0.47)$ & 0.106 & $1.68(0.90-3.14)$ & 0.038 & $2.18(1.05-4.56)$ & 0.140 & Exonic & Missense & A227P \\
\hline rs 434857 & $\mathrm{G}(0.47)$ & 0.106 & $1.68(0.90-3.14)$ & 0.038 & $2.18(1.05-4.56)$ & 0.140 & Exonic & Synonymous & P32P \\
\hline rs 445784 & $\mathrm{~T}(0.47)$ & 0.106 & $1.68(0.90-3.14)$ & 0.038 & $2.18(1.05-4.56)$ & 0.140 & Exonic & Synonymous & V194V \\
\hline rs436525 & $\mathrm{A}(0.43)$ & 0.180 & $1.56(0.83-2.94)$ & 0.090 & $1.86(0.91-3.82)$ & 0.190 & Exonic & Synonymous & P500P \\
\hline \multicolumn{10}{|l|}{ ADAMTS13 } \\
\hline rs149586801 & $\mathrm{T}(0.07)$ & 0.011 & $0.26(0.07-0.78)$ & 0.012 & $0.18(0.05-0.69)$ & 0.679 & Intronic & - & - \\
\hline rs1055432 & A $(0.17)$ & 0.282 & $0.64(0.29-1.37)$ & 0.080 & $0.48(0.22-1.09)$ & 0.755 & Exonic & Synonymous & $\mathrm{T} 1407 \mathrm{~T}$ \\
\hline \multicolumn{10}{|l|}{ ADAMTS14 } \\
\hline rs4747097 & $\mathrm{T}(0.38)$ & 0.165 & $1.61(0.88-2.98)$ & 0.078 & $1.83(0.93-3.56)$ & 0.644 & Intronic & - & - \\
\hline
\end{tabular}

Significant data $(P<0.05)$ are indicated in bold

$M M P$ matrix metalloproteinase, TIMP tissue inhibitors of metalloproteinase, ADAMTS, a disintegrin and metalloproteinase with thrombospondin motif, $S N P$ single-nucleotide polymorphism, $C I$ confidence interval, $O R$ odds ratio, $H W E$ Hardy-Weinberg equilibrium, $A A$ amino acid, $T$ threonine, $A$ alanine, $P$ proline, $V$ valine, $S$ serine, $H$ histidine

${ }^{a}$ Adjusted by pregnancy and parity

MMP3 can activate other MMPs, such as MMP1 [25]. TIMPs are specific inhibitors of MMPs and key regulator for their activities. The proper proportion of MMPs and TIMPs can maintain the dynamic balance of ECM and thus may maintain the stability of the supporting structure of the pelvic floor.

The role of MMPs in the development of POP has been primarily explored in their expression changes as well as their susceptible loci for POP. Previous analysis demonstrated that women with POP showed higher expression levels of MMP1 and MMP8 compared with the control women [26]. The expression of MMP2 and MMP9 were higher in the uterosacral ligament in women with POP [27]. For the analysis of MMP polymorphisms, limited MMPs have been explored. Since the sequencing in this study did not involve the promoter regions of $M M P 1,3$, and 9 in our target region sequencing approach, we did not verify our data in relation to the previous polymorphisms in the promoter regions of $M M P 1,3$, and 9. However, we compared our results to the previous $M M P 9$ and 10 studies [12-14]. Chen et al. [12] conducted a case-control associated study evaluating three MMP9 SNPs, rs3918242, rs17576, and rs2250889, and found that rs $17576 \mathrm{G}$ allele was a risk site for POP. The authors recruited 92 POP patients at POP-Q stage $\geq$ II and 152 control women at stage $0-\mathrm{I}$ of Taiwanese women. Wu et al. [13] additionally detected $8 M M P 9$ SNPs and found rs3918253 and rs3918256 associated with POP in nonHispanic white women in 239 cases with stages III and IV and 197 controls with stages $0-$ I. In our study, we did not find the previously reported MMP9 SNPs rs3918253 and rs3918256 by Wu et al. [13] in significant association with POP. Chen et al. [12] reported rs17576 was significantly associated with POP; however, Wu et al. [13] and our study both obtained the opposite result. Moreover, for other previously detected MMP9 SNPs rs3918242, rs2250889, rs3918278, rs2274755, rs17577, rs2236416, and rs3787268 by Wu et al. 
A

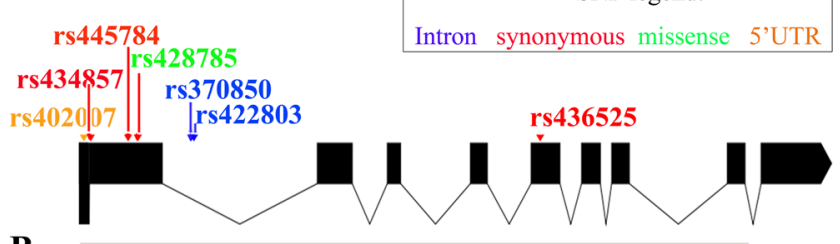

B

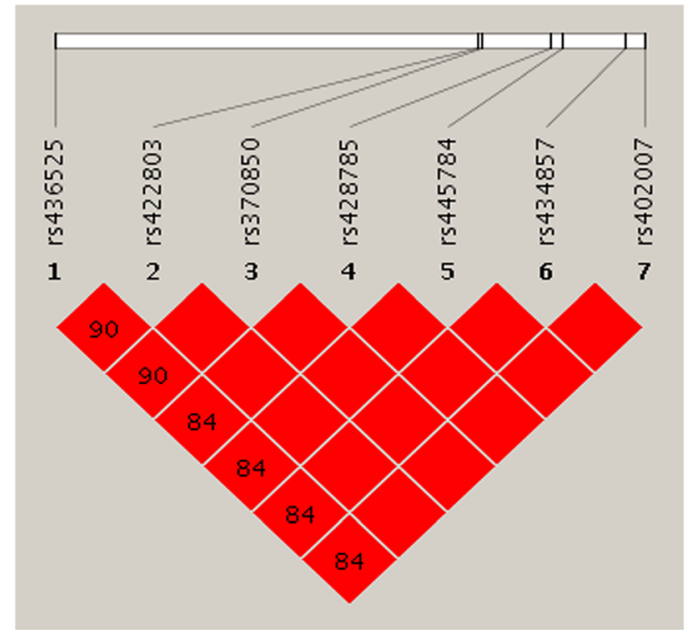

Fig. 1 ADAMTS1 gene structure, indication, and the linkage disequilibrium (LD) of the SNPs. (A) Gene structure of ADAMTS1. Exons are presented in the vertical black regions. SNPs with a significant or suggestively significant association with POP are indicated by arrows in different colors: synonymous (red), missense (green), introns (blue), and 5'UTR (orange). (B) LD graphics for ADAMTS1 SNPs with $\mathrm{r}^{2}$ in each of the boxes. Two SNPs showed greater correlation when the $r^{2}$ value was closer to 1 . Boxes with no values meant $r^{2}=1$ for perfect $L D$

and Chen et al., we confirmed that these SNPs also showed no association with POP in our study. Besides validating the previous study, we additionally identified a MMP9 SNP rs3918254 that had a suggestively significant association with POP, which was an intronic SNP. Wang et al. [14] detected a higher MMP10 serum level in the POP group than in the control group and also found MMP10 SNP rs17435959 genotype $\mathrm{G} / \mathrm{C}$ was distributed differentially between 91 women with POP at stage $\geq$ II and 172 control women at stage 0-I of Chinese ancestry. However, we did not find a significant association in the MMP10 gene in our study. The inconsistency of these studies may partially be attributed to the differences in the recruitment criteria and the characteristics of the women, for example, the race, age, BMI, parity, etc., which were key influencing factors for POP, or in the detection methods.

Studies on other MMP polymorphisms have not been conducted in POP. In this study, besides MMP9 and MMP10, we have sequenced multiple MMP genes and aimed to find the association between these genes and POP. However, we did not observe any positive associations among $M M P 1,2,3,8,10$, and POP, respectively. Additionally, we have found two new MMP13
SNPs, rs3758853 and rs78356340, which showed borderline significant associations with POP and may have a risk effect for POP.

There were few studies focusing on TIMP family genes and POP. TIMP2 expression level in the POP group was lower than that in the control group [27]. TIMP3 also showed lower levels in the uterosacral ligament in women with POP compared to non-POP women [28]. In our study, we sequenced TIMP1, 2, 3, and 4 genes for the first time. We found a significant association between a TIMP2 SNP rs2277698 and POP. Our data suggested that it may show a protective effect for POP development. We also reported a TIMP3 SNP rs9862 and a TIMP4 SNP rs10433537 that had a borderline association with POP, which should be further investigated in future research.

The ADAMTS protease family plays crucial roles in ECM remodeling and tissue morphogenesis as well as in inflammation and other physiological and pathological processes [29]. This family consists of 19 members which belong to different subgroups. For example, ADAMTS1 and 8 are the aggrecanases or proteoglycanases [30], ADAMTS2, 3, and 14 are the N-propeptidases of the procollagen [31], and ADAMTS13 is the well-recognized von-Willebrand factor proteinase [32]. ADAMTSs participate in the synthesis of ECM by their effects on the N-propeptidases of the procollagens and in the modification of ECM proteoglycans by their aggrecanase function. However, several studies on ADAMTSs have been performed in relation to the development of POP. One of the ADAMTS members, ADAMTS16, was identified as genome wide suggestive in a genome-wide association study (GWAS) on urgency urinary incontinence (UUI) [33], indicating the involvement of ADAMTS16 in this pelvic floor dysfunction. In our study, we found a significant association among ADAMTS1 SNPs rs370850, rs422803, rs402007, rs428785, rs434857, and rs445784 and POP, which may be risk factors related to POP, and a significant association between ADAMTS13 SNP rs149586801 and POP, which showed potential protective effects for this disease. We also found three additional SNPs, ADAMTS1 SNP rs436525, ADAMTS13 SNP rs1055432, and ADAMTS14 SNP rs 4747097, which have suggestively significant associations with POP. Additionally, ADAMTS1 SNP rs428785 is a missense SNP and can lead to a substitution of alanine to proline, which might exhibit a benign and tolerant effect predicted by PolyPhen-2 and SIFT. Therefore, ADAMTS1, ADAMTS13, and ADAMTS14 could be novel candidate genes for POP, and this needs further validation.

Our study had several strengths. As previous studies were few and focused on certain limited SNPs of a gene, we sequenced several MMP, TIMP, and ADAMTS family genes by target region sequencing technique to assess the entire genes with their coding regions. Second, we not only validated the data of the previously identified SNPs in MMP9 and 10, but 
also analyzed the association between $M M P 1,2,3,8$, and 13 and POP for the first time. Additionally, the polymorphisms of TIMP and some ADAMTS family genes have been first studied in POP, suggesting new susceptible SNPs. Third, we focused on unrelated Chinese women from mainland China in this candidate gene association study, choosing extreme symptoms of severe POP with POP-Q stages III and IV, tying to improve the detection of the potential variants.

However, the main limitation of our study was the small sample size. Since our nationwide epidemiological survey in mainland China found that the prevalence of POP at stages III and IV was $2.04 \%$ [34], this made it quite difficult to select women under this stage limitation within a certain period of time. Thus, power may be limited in this study because of the relatively small sample size. Although we accepted all variants with a minor allele frequency of 5\%, variants identified as being significant or suggestive had a much higher allele frequency than 5\%. If we only consider the variants of significant associations (allele frequencies ranging from $0.07-0.47$, OR ranging from 0.18-3.71), the power can make our data use a reference to some extent according to the different OR value of these SNPs. Hypotheses regarding the pathophysiology of POP include abnormal synthesis or degradation of ECM, and we provided new evidence on MMP, TIMP, and ADAMTS polymorphisms for future investigation of the involvement of these candidate genes in the etiology of POP. The genetic contributions to POP remain poorly understood. Additional work needs to be done to provide further validation of POP predisposition variants in a variety of different populations to establish the role of these genes in the pathogenesis of prolapse [5].

\section{Conclusion}

MMP, TIMP and ADAMTS family genes are crucial for ECM synthesis, modification, and metabolism. In this study, we provide initial evidence that the genetic variants in these genes may have an association with POP. We identified several susceptible SNPs in the MMP9 and 13, TIMP2, 3, and 4 and ADAMTS1, 13, and 14 genes in Chinese groups, preliminarily revealing the underlying mechanisms for the pathophysiology of this common disease.

\footnotetext{
Abbreviations $P O P$, Pelvic organ prolapse; COL1A1, Collagen type I alpha; COL3A1, Collagen type III alpha 1; LAMC1, Laminin gamma-1; $M M P$, Matrix metalloproteinase; ECM, Extracellular matrix; TIMP, Tissue inhibitors of metalloproteinase; ADAMTS, A disintegrin and metalloproteinase with thrombospondin motif; $S N P$, Single-nucleotide polymorphism; $P O P-Q$, Pelvic organ prolapse quantification; $B W A$, BurrowsWheeler Aligner; $M A F$, Minor allele frequency; $O R$, Odds ratio; $C I$, Confidence interval; $L D$, Linkage disequilibrium; $B M I$, Body mass index; $G W A S$, Genome-wide association study; UUI, Urgency urinary incontinence
}

Supplementary Information The online version contains supplementary material available at https://doi.org/10.1007/s00192-021-04917-5.

Funding This study was supported by the National Natural Science Foundation of China (81830043) and the CAMS Innovation Fund for Medical Sciences (CIFMS-2017-I2M-1-002).

\section{Declarations}

Ethics approval This study was approved and guided by the ethical committee of the Peking Union Medical College Hospital. Informed consent was obtained from all participants.

\section{Conflicts of interest None.}

Competing interests The authors declare that they have no competing interests.

Financial disclaimer None.

Open Access This article is licensed under a Creative Commons Attribution 4.0 International License, which permits use, sharing, adaptation, distribution and reproduction in any medium or format, as long as you give appropriate credit to the original author(s) and the source, provide a link to the Creative Commons licence, and indicate if changes were made. The images or other third party material in this article are included in the article's Creative Commons licence, unless indicated otherwise in a credit line to the material. If material is not included in the article's Creative Commons licence and your intended use is not permitted by statutory regulation or exceeds the permitted use, you will need to obtain permission directly from the copyright holder. To view a copy of this licence, visit http://creativecommons.org/licenses/by/4.0/.

\section{References}

1. Jelovsek JE, Maher C, Barber MD. Pelvic organ prolapse. Lancet. 2007;369(9566):1027-38.

2. Li Z, Xu T, Li Z, Gong J, Liu Q, Wang Y, et al. An epidemiologic study of pelvic organ prolapse in postmenopausal women: a population-based sample in China. Climacteric. 2019;22(1):79-84.

3. Barber MD. Pelvic organ prolapse. BMJ. 2016;354:i3853.

4. Feiner B, Fares F, Azam N, Auslender R, David M, Abramov Y. Does COLIA1 SP1-binding site polymorphism predispose women to pelvic organ prolapse? Int Urogynecol J Pelvic Floor Dysfunct. 2009;20(9):1061-5.

5. Allen-Brady K, Chua JWF, Cuffolo R, Koch M, Sorrentino F, Cartwright R. Systematic review and meta-analysis of genetic association studies of pelvic organ prolapse. Int Urogynecol J. 2021. https://doi.org/10.1007/s00192-021-04782-2.

6. Li L, Sun Z, Chen J, Zhang Y, Shi H, Zhu L. Genetic polymorphisms in collagen-related genes are associated with pelvic organ prolapse. Menopause. 2020;27(2):223-9.

7. Li L, Kang J, Zhang Y, Mao M, Yang Y, Lang J, et al. LAMC1, LAMA2 and LAMA3 gene polymorphisms and the risk for severe pelvic organ prolapse. Sci Bull. 2019;7(64):466-8.

8. Karachalios C, Bakas P, Kaparos G, Demeridou S, Liapis I, Grigoriadis C, et al. Matrix metalloproteinase-3 gene promoter polymorphisms: a potential risk factor for pelvic organ prolapse. Biomed Rep. 2016;5(3):337-43.

9. Skorupski P, Jankiewicz K, Miotla P, Marczak M, KulikRechberger B, Rechberger T. The polymorphisms of the MMP-1 
and the MMP-3 genes and the risk of pelvic organ prolapse. Int Urogynecol J. 2013;24(6):1033-8.

10. Ferrari MM, Rossi G, Biondi ML, Vigano P, Dell'utri C, Meschia M. Type I collagen and matrix metalloproteinase 1, 3 and 9 gene polymorphisms in the predisposition to pelvic organ prolapse. Arch Gynecol Obstet. 2012;285(6):1581-6.

11. Skorupski P, Miotla P, Jankiewicz K, Rechberger T. MMP-1 and MMP-3 gene encoding polymorphism and the risk of the development of pelvic organ prolapse and stress urinary incontinence. Ginekol Pol. 2010;81(8):594-9.

12. Chen HY, Lin WY, Chen YH, Chen WC, Tsai FJ, Tsai CH. Matrix metalloproteinase-9 polymorphism and risk of pelvic organ prolapse in Taiwanese women. Eur J Obstet Gynecol Reprod Biol. 2010;149(2):222-4.

13. Wu JM, Visco AG, Grass EA, Craig DM, Fulton RG, Haynes C, et al. Matrix metalloproteinase- 9 genetic polymorphisms and the risk for advanced pelvic organ prolapse. Obstet Gynecol. 2012;120(3):587-93.

14. Wang H, Zhang ZQ, Wang SZ. Association of matrix metalloproteinase-10 polymorphisms with the susceptibility to pelvic organ prolapse. J Minim Invasive Gynecol. 2015;22(6S):S68.

15. Allen-Brady K, Cannon-Albright LA, Farnham JM, Norton PA. Evidence for pelvic organ prolapse predisposition genes on chromosomes 10 and 17. Am J Obstet Gynecol. 2015;212(6):771.e1-7.

16. Kelwick R, Desanlis I, Wheeler GN, Edwards DR. The ADAMTS (A Disintegrin and Metalloproteinase with Thrombospondin motifs) family. Genome Biol. 2015;16:113.

17. Alarab M, Kufaishi H, Lye S, Drutz H, Shynlova O. Expression of extracellular matrix-remodeling proteins is altered in vaginal tissue of premenopausal women with severe pelvic organ prolapse. Reprod Sci. 2014;21(6):704-15.

18. Dos Santos RGM, Pepicelli FCA, Batista NC, de Carvalho CV, Bortolini MAT, Castro RA. Collagen XVIII and LOXL-4 polymorphisms in women with and without advanced pelvic organ prolapse. Int Urogynecol J. 2018;29(6):893-8.

19. Fuchsberger C, Flannick J, Teslovich TM, Mahajan A, Agarwala $\mathrm{V}$, Gaulton KJ, et al. The genetic architecture of type 2 diabetes. Nature. 2016;536(7614):41-7.

20. Li K, Tay FR, Yiu CKY. The past, present and future perspectives of matrix metalloproteinase inhibitors. Pharmacol Ther. 2020;207: 107465.

21. Alaseem A, Alhazzani K, Dondapati P, Alobid S, Bishayee A, Rathinavelu A. Matrix metalloproteinases: a challenging paradigm of cancer management. Semin Cancer Biol. 2019;56:100-15.

22. Vandenbroucke RE, Libert C. Is there new hope for therapeutic matrix metalloproteinase inhibition? Nat Rev Drug Discov. 2014;13(12):904-27.
23. Allan JA, Docherty AJ, Barker PJ, Huskisson NS, Reynolds JJ, Murphy G. Binding of gelatinases A and B to type-I collagen and other matrix components. Biochem J. 1995;309(Pt 1):299-306.

24. Patterson ML, Atkinson SJ, Knauper V, Murphy G. Specific collagenolysis by gelatinase A, MMP-2, is determined by the hemopexin domain and not the fibronectin-like domain. FEBS Lett. 2001;503(2-3):158-62.

25. Visse R, Nagase H. Matrix metalloproteinases and tissue inhibitors of metalloproteinases: structure, function, and biochemistry. Circ Res. 2003;92(8):827-39.

26. $\mathrm{Hu} \mathrm{Y,} \mathrm{Wu} \mathrm{R,} \mathrm{Li} \mathrm{H,} \mathrm{Gu} \mathrm{Y,} \mathrm{Wei} \mathrm{W.} \mathrm{Expression} \mathrm{and} \mathrm{significance} \mathrm{of}$ metalloproteinase and collagen in vaginal wall tissues of patients with pelvic organ prolapse. Ann Clin Lab Sci. 2017;47(6):698-705.

27. Liu C, Wang Y, Li BS, Yang Q, Tang JM, Min J, et al. Role of transforming growth factor betal in the pathogenesis of pelvic organ prolapse: a potential therapeutic target. Int J Mol Med. 2017;40(2):347-56.

28. Tola EN, Koroglu N, Yildirim GY, Koca HB. The role of ADAMTS-2, collagen type-1, TIMP-3 and papilin levels of uterosacral and cardinal ligaments in the etiopathogenesis of pelvic organ prolapse among women without stress urinary incontinence. Eur J Obstet Gynecol Reprod Biol. 2018;231:158-63.

29. Zhong S, Khalil RA. A Disintegrin and Metalloproteinase (ADAM) and ADAM with thrombospondin motifs (ADAMTS) family in vascular biology and disease. Biochem Pharmacol. 2019;164: 188-204.

30. Choi GC, Li J, Wang Y, Li L, Zhong L, Ma B, et al. The metalloprotease ADAMTS8 displays antitumor properties through antagonizing EGFR-MEK-ERK signaling and is silenced in carcinomas by CpG methylation. Mol Cancer Res. 2014;12(2):228-38.

31. Bekhouche $\mathrm{M}$, Colige $\mathrm{A}$. The procollagen $\mathrm{N}$-proteinases ADAMTS2, 3 and 14 in pathophysiology. Matrix Biol. 2015;4446:46-53.

32. Akiyama M, Takeda S, Kokame K, Takagi J, Miyata T. Crystal structures of the noncatalytic domains of ADAMTS13 reveal multiple discontinuous exosites for von Willebrand factor. Proc Natl Acad Sci U S A. 2009;106(46):19274-9.

33. Richter HE, Whitehead N, Arya L, Ridgeway B, Allen-Brady K, Norton $\mathrm{P}$, et al. Genetic contributions to urgency urinary incontinence in women. J Urol. 2015;193(6):2020-7.

34. Pang H, Zhang L, Han S, Li Z, Gong J, Liu Q, et al. A nationwide population-based survey on the prevalence and risk factors of symptomatic pelvic organ prolapse in adult women in China- a pelvic organ prolapse quantification system-based study. BJOG. 2021;128(8):1313-1323.

Publisher's note Springer Nature remains neutral with regard to jurisdictional claims in published maps and institutional affiliations. 